\title{
Taming fluorescent dyes with cucurbituril
}

\author{
Werner M. Nau ${ }^{1, \dagger}$ and Jyotirmayee Mohanty ${ }^{1,2, \neq}$ \\ ${ }^{1}$ School of Engineering and Science, International University Bremen, Campus Ring 1, D-28759 Bremen, Germany \\ ${ }^{2}$ Radiation Chemistry \& Chemical Dynamics Division, Bhabha Atomic Research Centre, Mumbai, India
}

\begin{abstract}
The potential of the supramolecular host molecule cucurbit[7]uril to serve as a stabilizing additive and enhancement agent was investigated for the following dyes in aqueous solution: rhodamine 6G, rhodamine 123, tetramethylrhodamine, cresyl violet, fluorescein, coumarin 102, pyronin B, pyronin Y, two cyanine 5 and one cyanine 3 derivative, and IR140 as well as IR144. For most cationic dyes photostabilization was established, and a pronounced thermal stabilization due to deaggregation and solubilization was observed for the xanthene dyes. The advantageous effects are attributed to the formation of inclusion complexes with different photophysical and photochemical properties. The complexation is accompanied by spectral shifts characteristic for the inclusion in a less polar environment, while the fluorescence quantum yields as well as the brightness show an increase, with few exceptions. As a consequence of the low polarizability inside the cucurbituril cavity, the fluorescence lifetimes of the included dyes increase substantially and systematically. Applications of the new photostabilizing additive for dye lasers, for prolonged storage of dye solutions, in scanning confocal microscopy, and fluorescence correlation spectroscopy are discussed.
\end{abstract}

\section{INTRODUCTION}

Supramolecular complexation of chromophoric guest molecules by macrocyclic hosts can affect their fluorescent properties as a consequence of an altered microenvironment [1]. The classical example are aromatic guests like anilinonaphthalenesulfonates, which may be nonfluorescent or weakly fluorescent in water, but which become strongly fluorescent upon addition of a cyclodextrin (CD, structure below) as host [2, 3]; the enhancement is commonly understood in terms of the relocation of the guest into the more hydrophobic environment inside the $\mathrm{CD}$, such that the fluorescence properties resemble those observed in a less polar solvent. Cucurbit[n]urils are another class of host molecules capable of encapsulating guests in a hydrophobic cavity $[4,5]$. Although their supramolecular chemistry has recently been intensively investigated, relatively few investigations have dealt with photochemical investigations like the effect on fluorophores [6-13]. In the present study, we have investigated the complexation behavior of cucurbit[7]uril (CB7, structure below) [5] with several practically important fluorescent dyes (Chart 1): rhodamine 6G (Rh6G), tetramethylrhodamine (TMR), rhodamine 123 (Rh123), pyronin Y (PyY), pyronin B (PyB), coumarin 102 (C102), cresyl violet (CV), the cyanine derivatives $\mathrm{Cy}^{\prime}{ }^{\prime}, \mathrm{Cy} 5^{\prime}$, and $\mathrm{Cy} 5$, fluorescein (FL, an anionic dye) and the infrared dyes IR140 and IR144.

\section{RESULTS AND DISCUSSION}

Fluorescent dyes of the xanthene, coumarine, oxazine, and cyanine type have wide-spread technological,

\footnotetext{
${ }^{\dagger}$ E-mail: w.nau@iu-bremen.de

‡E-mail: jyotim@magnum.barc.ernet.in
}

scientific, and medicinal applications, e.g., for single molecule detection [14, 15], fluorescence labelling $[16,17]$, dye lasers [18], conversion and storage of solar energy [19], fluorescence-based assays [20], and staining of cells and antitumor agents [21]. In the search for the ultimate fluorescent dyes with highest photostability and brightness in water as environmentally benign and biologically relevant solvent, strategies involving stabilizing, solubilizing, deaggregating, and enhancing additives have become popular. The use of organic solvents is the simplest approach, but their range is often restricted to protic/alcoholic solvents due to the ionic nature of the dyes, they are incompatible with biologically or environmentally relevant applications, and their use on large scales, e.g., in the recycling of laser dye solutions, is frequently discouraged by economical and environmental considerations.

The strategy of micellation of dyes [22-25], which facilitates solubilization and assists deaggregation, requires large amounts of detergents to be added, and frequently leads to undesirable effects in regard to photostability or, in the case of dye lasers, adverse thermooptic and light scattering solvent properties. The strategy of host-guest complexation, on the other hand, has been explored in several case studies for CDs [26, 27] and more recently water-soluble calixarenes as macrocyclic hosts [28]. While some improvements were observed, mainly due to a deaggregating influence [29], the addition of CDs presents no universal approach, and quite frequently displays negative effects, in particular a reduced brightness of those xanthene dyes which already have very high quantum yields without additive [30]. Importantly, the binding constants which CDs exhibit with the fluorescent dyes are generally small, on the order of $10^{2}-10^{3} \mathrm{M}^{-1}[28,30]$, which 


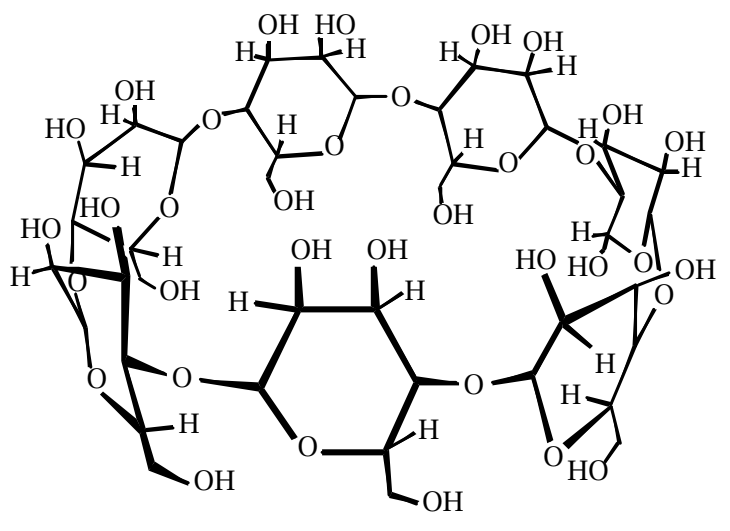

$\beta$-cyclodextrin $(\beta$-CD)

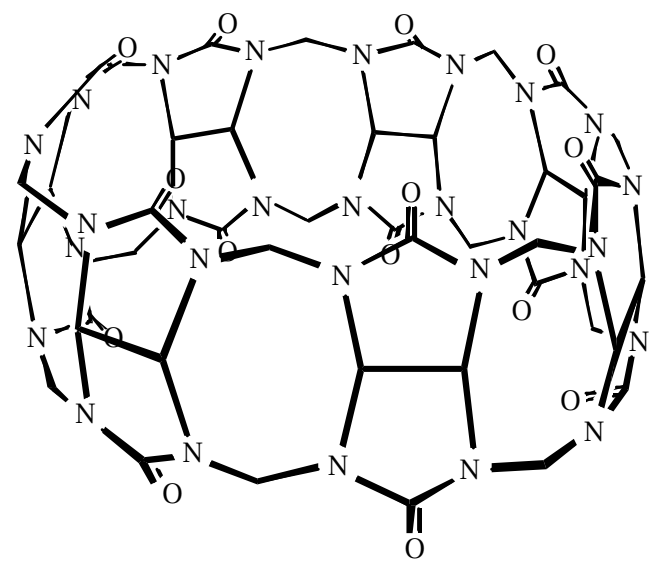

cucurbit[7]uril (CB7) would require an excessive host concentration to ensure nearly-quantitative binding. To stabilize the complexes, a strategy employing rotaxane formation with CDs as threading macrocycles has been explored for cyanine derivatives [31], but this appears not viable for routine dye use.

We now document a combination of several beneficial effects of $\mathrm{CB} 7$ on the fluorescence properties of several cationic dyes. Although most of the investigated dyes (Chart 1) are already structurally optimized with respect to their fluorescent properties in water (except for the IR dyes), CB7 exhibits a range of desirable effects, most importantly an enhancement of thermal and photochemical stability, which is accompanied, depending on the dye, by an enhanced brightness. These desirable properties are related to the distinct nature of the CB7 cavity, in particular its exceptionally low chemical reactivity and low polarizability.

Addition of CB7 [10, 11, 32] to solutions of most cationic fluorescent dyes results in the immediate formation of inclusion complexes, as evidenced by the concomitant changes of the photophysical parameters of the fluorescent dyes (Table 1) and the complexationinduced upfield shifts in the ${ }^{1} \mathrm{H}$ NMR spectra of the chromophoric guests, which are well-known for cucurbituril complexation [4, 33]. An independent indicator for complex formation is the 2-3 times reduced diffusion coefficient of the dye upon complexation of CB7 [34] as measured by fluorescence correlation spectroscopy (FCS) [35, 36]. The spectral shifts can be conveniently employed to derive the binding stoichiometry of the dyes with CB7. In general, the corresponding titration plots (see below) were consistent with the formation of $1: 1$ complexes. In the case of $\mathrm{Cy}^{\prime}, \mathrm{CV}$, and PyY a 2 : 1 complexation, in which two CB7 host molecules associate with a single dye molecule, was indicated. Note that cucurbiturils have cation receptor properties; this facilitates an efficient complexation of the cationic dyes, which represent by far the dominant class of dyes. $p$-Sulfonatocalix $[n]$ arenes are cation receptors as well and do in fact bind some fluorescent dyes equally strong ( $>10^{4} \mathrm{M}^{-1}$ for $n=6,8$ ), but do not show the advantageous effects of $\mathrm{CB} 7$, e.g., they quench the fluorescence, presumably due to their high chemical reactivity, e.g., a lower oxidation potential [28].

The presence of a cationic charge in the molecule alone does not ensure binding by $\mathrm{CB} 7$, while anionic centers result in low binding, unless they are remotely tethered and not adjacent to the chromophoric, mostly nitrogen-bearing, aromatic unit itself. For example, the popular Cy5 dye, which carries a sulfonate group directly on the aromatic ring, does not undergo significant binding with $\mathrm{CB} 7$, while the presently investigated derivatives $\mathrm{Cy}^{\prime}$ and $\mathrm{Cy}^{\prime}$, which carry a remotely tethered sulfonate group, do form complexes with CB7. Expectedly, the anionic dye FL does not form a complex with CB7 either. These "inactive" dyes (Cy5 and FL) were conveniently used as negative controls in the various experiments. The limits of CB7 become also obvious for the IR dyes, which are also special cases, since they are either insoluble (IR140) or nonfluorescent (IR144) in water. The former remained insoluble in the presence of CB7, while the latter did form an inclusion complex with CB7 ( $K$ ca. $10^{4} \mathrm{M}^{-1}$ ), as evidenced by UV absorption shifts, but remained nonfluorescent, although IR144 emits in organic solvents.

Having established the formation of inclusion complexes for most cationic dyes, we now turn to the detailed advantageous features of the additive $\mathrm{CB} 7$ on their fluorescent properties. First of all, the binding constants, as obtained by UV-Vis spectrophotometric and steady-state fluorescence titrations, are very large $\left(10^{4}-10^{5} \mathrm{M}^{-1}\right.$ for 1 : 1 complex formation). This allows the use of low $\mathrm{mM} \mathrm{CB} 7$ concentrations to ensure a virtually quantitative (> 90\%) complexation at the relevant $\mathrm{nM}-\mu \mathrm{M}$ fluorescent dye concentrations, 
Chart 1 (counter ions not shown)<smiles>[R12][R17]=c1cc2c(cc1[R])=C(c1ccccc1C([R])=O)c1cc([R])c([Y10])cc1O2</smiles>

Rh6G : $\mathrm{R}^{1}=\mathrm{H}, \mathrm{R}^{2}=\mathrm{Et}, \mathrm{R}^{3}=\mathrm{Me}, \mathrm{R}^{4}=\mathrm{Et}$ TMR : $\mathrm{R}^{1}=\mathrm{R}^{2}=\mathrm{Me}, \mathrm{R}^{3}=\mathrm{H}, \mathrm{R}^{4}=\mathrm{H}$ Rh123: $\mathrm{R}^{1}=\mathrm{R}^{2}=\mathrm{H}, \mathrm{R}^{3}=\mathrm{H}, \mathrm{R}^{4}=\mathrm{Et}$<smiles>Cc1cc(=O)oc2c3c4c(cc12)CCCN4CCC3</smiles>

C102

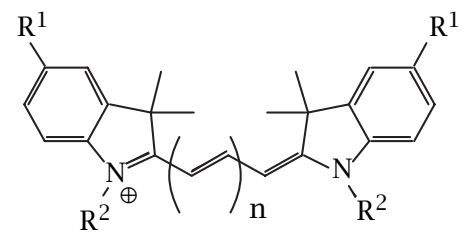

$\mathrm{Cy}^{\prime}: \mathrm{R}^{1}=\mathrm{H}, \mathrm{R}^{2}=\left(\mathrm{CH}_{2}\right)_{4} \mathrm{SO}_{3}{ }^{-}, \mathrm{n}=1$

$\mathrm{Cy}^{\prime}: \mathrm{R}^{1}=\mathrm{H}, \mathrm{R}^{2}=\left(\mathrm{CH}_{2}\right)_{4} \mathrm{SO}_{3}{ }^{-}, \mathrm{n}=2$

Cy5 : $\mathrm{R}^{1}=\mathrm{SO}_{3}{ }^{-}, \mathrm{R}^{2}=\mathrm{Et}, \mathrm{n}=2$<smiles>[R10]N=c1ccc2cc3ccc([N+]#[R10])cc3oc-2c1</smiles>

PyY $: \mathrm{R}=\mathrm{Me}$

$\mathrm{PyB}: \mathrm{R}=\mathrm{Et}$<smiles>Nc1ccc2nc3c4ccccc4c(=[NH2+])cc-3oc2c1</smiles>

CV<smiles>O=C([O-])c1ccccc1-c1c2ccc(=O)cc-2oc2cc([O-])ccc12</smiles>

FL

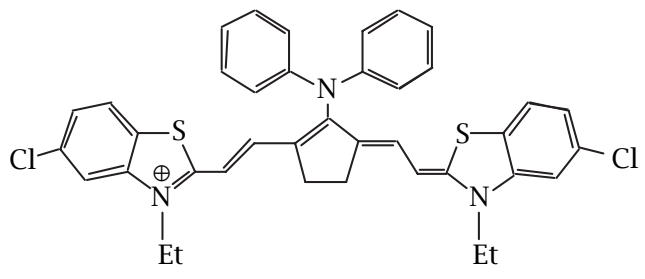

IR140

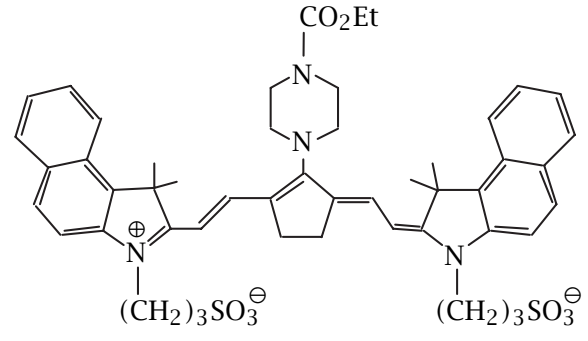

IR144 except for Rh123, which shows a weaker binding (ca. $1000 \mathrm{M}^{-1}$ ). Examples of UV-Vis titration plots are shown in Figure 1 for TMR and Rh123.

Second, complexation with CB7 increases the brightness of most dyes, defined here as product of the extinction coefficient at the absorption maximum and the fluorescence quantum yield (Table 1), which is a decisive feature for practical applications. The increased brightness can generally be traced back to a larger quantum yield (up to a factor of 2). The effects on the extinction coefficients are less systematic and result in a band broadening or sharpening upon complexation, but not in an overall change for the oscillator strength (integrated absorption band). The latter remains constant upon complexation, as expected for a strongly allowed transition [12]. It should be noted that the increase of the quantum yield is for some dyes spectacular, e.g., for $\mathrm{PyB}$ and the red-emitting $\mathrm{Cy} 5^{\prime}$, larger than what could readily be achieved by the selection of different solvents [30], and similar to the improvement resulting from structural optimization (cf. other Cy5 derivative). The more efficient fluorescence emission inside $\mathrm{CB} 7$ is presumably due to a combination of factors, in particular the inclusion in a rigid confined environment, which may slow down intramolecular deactivation pathways, e.g., by preventing intramolecular rotation about the xanthene-amino bond of PyB [37, 38] or by isomerization of the polymethine linkage in Cy5 
Table 1. Photophysical parameters of fluorescent dyes with and without $1 \mathrm{mM} \mathrm{CB} 7$ in $\mathrm{H}_{2} \mathrm{O}$.

\begin{tabular}{|c|c|c|c|c|c|c|c|c|}
\hline \multicolumn{2}{|c|}{$\begin{array}{l}\text { Fluorescent dye } \\
\text { (OD ca. } 0.10)\end{array}$} & \multirow{3}{*}{$\begin{array}{c}\begin{array}{c}\lambda_{\mathrm{abs}}^{\max } \\
/ \mathrm{nm}\end{array} \\
526 \\
535\end{array}$} & \multirow{3}{*}{$\begin{array}{c}\begin{array}{c}\lambda_{\mathrm{em}}^{\max } \\
/ \mathrm{nm}\end{array} \\
552 \\
555\end{array}$} & \multirow{3}{*}{$\begin{array}{c}\varepsilon /\left(10^{4} \mathrm{M}^{-1} \mathrm{~cm}^{-1}\right) \\
8.02 \\
9.24\end{array}$} & \multirow{3}{*}{\begin{tabular}{l}
\multicolumn{1}{c}{$\phi_{\mathrm{f}}$} \\
$0.89^{[\mathrm{c}]}$ \\
0.89
\end{tabular}} & \multirow{3}{*}{$\begin{array}{c}\text { brightness }^{[\mathrm{a}]} \\
7.14 \\
8.22\end{array}$} & \multirow{3}{*}{$\begin{array}{l}\tau_{\mathrm{f}} / \mathrm{ns} \\
4.08 \\
4.76\end{array}$} & \multirow{3}{*}{$\frac{\sqrt{\frac{k_{\mathrm{r}}^{\mathrm{CB} 7}}{k_{\mathrm{r}} \mathrm{H}_{2} \mathrm{O}}}}{0.93}$} \\
\hline & without CB7 & & & & & & & \\
\hline $\mathrm{Rh}_{6 G^{[\mathrm{b}]}}$ & with CB7 & & & & & & & \\
\hline \multirow{2}{*}{ TMR } & without CB7 & 553 & 577 & 8.78 & $0.28^{[\mathrm{d}]}$ & 2.46 & 2.15 & \multirow{2}{*}{0.84} \\
\hline & with CB7 & 559 & 582 & 7.48 & 0.38 & 2.84 & 4.16 & \\
\hline \multirow{2}{*}{ Rh123 } & without $\mathrm{CB} 7$ & 500 & 525 & 6.92 & $0.83^{[\mathrm{e}]}$ & 5.75 & 4.19 & \multirow{2}{*}{0.63} \\
\hline & with CB7 & 503 & 532 & 6.66 & 0.36 & 2.40 & 4.63 & \\
\hline \multirow{2}{*}{ PyY } & without CB7 & 546 & 565 & 13.2 & $0.47^{[\mathrm{f}]}$ & 6.20 & 1.69 & \multirow{2}{*}{0.81} \\
\hline & with CB7 & 544 & 568 & 13.1 & 0.63 & 8.25 & 3.44 & \\
\hline \multirow{2}{*}{ РyВ } & without CB7 & 552 & 569 & 9.41 & $0.36^{[\mathrm{f}]}$ & 3.39 & 1.19 & \multirow{2}{*}{0.86} \\
\hline & with CB7 & 556 & 571 & 9.93 & 0.70 & 6.95 & 3.10 & \\
\hline \multirow{2}{*}{ C102 } & without CB7 & 393 & 489 & 2.18 & $0.66^{[\mathrm{g}]}$ & 1.44 & 6.04 & \multirow{2}{*}{0.98} \\
\hline & with CB7 & 405 & 476 & 2.36 & 0.75 & 1.77 & 7.19 & \\
\hline \multirow{2}{*}{$\mathrm{CV}$} & without $\mathrm{CB} 7$ & 585 & 625 & 3.31 & $0.36^{[\mathrm{h}]}$ & 1.19 & 2.18 & \multirow{2}{*}{0.74} \\
\hline & with CB7 & 591 & 628 & 4.09 & 0.35 & 1.43 & 3.93 & \\
\hline \multirow{2}{*}{ Cy3' } & without CB7 & 545 & 560 & 12.0 & $0.04^{[\mathrm{i}]}$ & 0.48 & 0.46 & \multirow{2}{*}{0.77} \\
\hline & with CB7 & 559 & 571 & 10.7 & 0.03 & 0.32 & 0.58 & \\
\hline \multirow{2}{*}{ Су5 $5^{\prime}$} & without CB7 & 642 & 660 & 13.8 & $0.17^{[\mathrm{j}]}$ & 2.35 & 0.63 & \multirow{2}{*}{0.84} \\
\hline & with CB7 & 642 & 657 & 11.2 & 0.30 & 3.36 & 1.59 & \\
\hline \multirow{2}{*}{ Cy5 } & without CB7 & 647 & 663 & 25.0 & $0.27^{[\mathrm{k}]}$ & 6.75 & 1.23 & \\
\hline & with CB7 & \multicolumn{7}{|c|}{ no complexation with CB7 } \\
\hline \multirow{2}{*}{ FL (pH 9) } & without $\mathrm{CB} 7$ & 491 & 513 & 6.73 & $0.90^{[b]}$ & 6.06 & 4.37 & \\
\hline & with CB7 & \multicolumn{7}{|c|}{ no complexation with $\mathrm{CB} 7$} \\
\hline
\end{tabular}

[a] Calculated as $\varepsilon_{\max } \phi_{\mathrm{f}} /\left(10^{4} \mathrm{M}^{-1} \mathrm{~cm}^{-1}\right)$. [b] From ref. [52]. [c] From ref. [40]. [d] From ref. [57]. [e] Determined relative to FL (pH 9). [f] From ref. [30]. [g] From ref. [41]. [h] From ref. [58], value for $c=3.34 \times 10^{-6} \mathrm{M}$ was used. [i] Determined relative to TMR. [j] Determined relative to Cy5. [k] From ref. [59]; the vendor provides a very similar value of 0.28 .

[27, 39]. However, complexation by CB7 alone does not ensure a stronger fluorescence, as is seen from the negative result obtained for IR144 (see above).

Third, CB7 encapsulation causes unprecedented changes of the photophysical parameters of the fluorescent dyes. In most cases, there is a bathochromic shift in the absorption and fluorescence band (Table 1), which is characteristic for the immersion in a less polar environment [30, 40, 41]. The absorption maximum of Rh6G complexed by CB7, for example, is at the same position as in $n$-octanol $(\varepsilon=10.3)$ [40], and the spectral shifts upon complexation of Cy3' by CB7 are illustrated in Figure 2. Complexation results also in a reduced Stokes shift, e.g., by up to $5 \mathrm{~nm}$ for the xanthene dyes and $25 \mathrm{~nm}$ for $\mathrm{C} 102$, suggesting a smaller geometrical and solvent relaxation of the dye, as expected for inclusion in a less polar (and more confined) environment [40]. The spectral shifts and differential Stokes shifts could be of practical interest, for example, for tuneable dye laser applications. In this context, it should also be mentioned that the photophysical properties of the fluorescent dyes Cy3' and Cy5' are characterized for the first time herein.

Strikingly, the fluorescence lifetimes increase systematically for all investigated dyes $\left(\tau_{\mathrm{f}}\right.$ values in
Table 1). In some cases an increase by more than a factor of 2 is observed, which renders the fluorescence lifetimes the longest hitherto reported for the respective dyes in aqueous (and for most dyes also in organic) solution. This effect is nontrivial, since it is larger and generally opposite to the lifetime shortening observed in less polar organic solvents, as well as CDs [30, 40]. This is a consequence of the very low polarizability which any guest molecule experiences inside cucurbituril, which has been recently quantified by using a solvatochromic probe [10, 12]. In essence, the polarizability/refractive index inside the cavity lies close to that of the gas phase, such that the photophysical properties of immersed dyes should follow this trend [12]; unfortunately, we found no experimental values for the fluorescence lifetimes of the high-molecular weight ionic dyes in the gas phase to allow a direct comparison.

But what is the relationship between the refractive index and the fluorescence lifetime of a dye? According to the Strickler-Berg equation [42], which has its foundations on Einstein's spontaneous emission rate and Planck's black body radiation law, the radiative decay rate constant of a chromophore, which equals the fluorescence quantum yield divided by the fluorescence lifetime, increases with the square of the refractive 


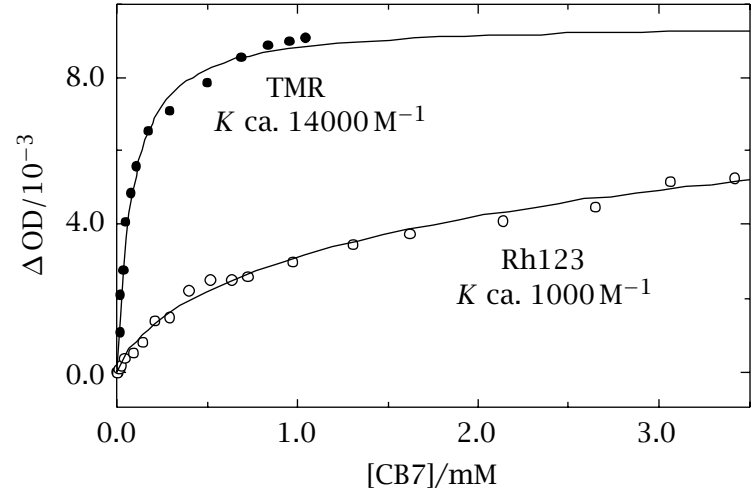

Figure 1. Representative titration plots for the visible absorptions of TMR (filled circles, ca. $1 \mu \mathrm{M}$ ) and Rh123 (open circles, ca. $1 \mu \mathrm{M}$ ) upon addition of increasing amounts of CB7.

index, such that small refractive indices result in slow radiative decay rates and expectedly longer fluorescence lifetimes. The longer fluorescence lifetimes of the fluorescent dyes are therefore fully consistent with the low polarizability inside the CB7 cavity [10]. In fact, the square root of the ratio of radiative decay rate constants (far right column in Table 1) multiplied with the refractive index of water provides a direct estimate of the refractive index inside the $\mathrm{CB} 7$ cavity. For the series of examined dyes a value of $1.10 \pm 0.12$ results (average for 9 complexing dyes), which falls nicely in between the refractive index of perfluorohexane (1.252, the organic solvent with lowest refractive index) and the gas phase ( $\equiv 1.000$ ). This projection is consistent with a previous estimate derived from solvatochromic effects on absorption spectra (1.187) [10, 12].

In view of the fact that the fluorescence rate inside CB7 is slowed relative to water (smaller radiative decay rate constant), it is the more surprising that the fluorescence quantum yield, which is the ratio of the radiative decay rate constant divided by the sum of radiative and radiationless decay rates, increases for several dyes (see above). It is therefore appropriate to argue that the fluorescence quantum yields are increased despite longer fluorescence lifetimes. The direct implication from this result is that the complexation by cucurbituril must decrease the radiationless decay rates by a larger extent than the radiative rates, e.g., by a factor of 2 for TMR. This demonstrates that the supramolecular host provides an active protection towards radiationless decay, e.g., by partial protection from water, which is well known to facilitate radiationless decay of xanthene dyes [40]. It is important to note that long fluorescence lifetimes of strong fluorescent dyes are quintessential for time-resolved fluorescence-based assays, which become nowadays of increasing importance for high-throughput screening in the context of drug

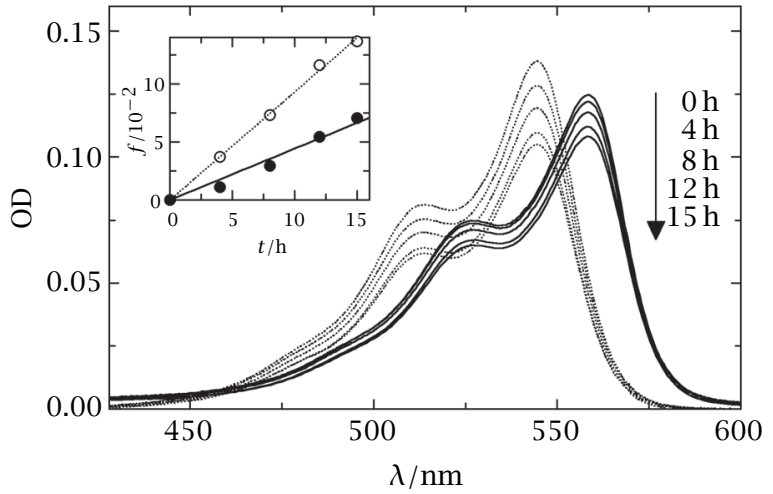

Figure 2. Photobleaching of $\mathrm{Cy}^{\prime}$ (ca. $1 \mu \mathrm{M}$ ) in aerated water in the absence (dotted lines) and presence (solid lines) of $1 \mathrm{mM} \mathrm{CB} 7$ followed through the decrease of the visible absorption with increasing time of white light irradiation in a photoreactor. The inset shows the plots and correlation lines for the characteristic function, $f=$ $\log \left(\left[10^{A} 0_{-1}\right] /\left[10^{A}-1\right]\right)$, versus irradiation time for the determination of the relative photobleaching quantum yield, cf. Experimental Section. The spectra at $t=0 \mathrm{~h}$ illustrate the spectral shifts caused by addition of $\mathrm{CB} 7$.

delivery [43, 44]. In short, the addition of CB7 to labelled peptides [11] or oligonucleotides [45] could lead to a prolonged fluorescence lifetime, which is more readily differentiated from background luminescence and scattered light, thereby resulting in dramatic improvements of the signal-to-noise ratio even if the absolute lifetime increase is moderate (50-100\%) [11]. The modulation of fluorescence lifetimes by additives could be additionally employed to increase the contrast in the emerging technique of fluorescence lifetime imaging microscopy (FLIM) [46].

As an additional asset, the administration of CB7 reduces photobleaching and therefore enhances the photostability of several fluorescent dyes towards chemical decomposition, in line with the observed reduction in radiationless deactivation rates (see above). Several additives have been recommended and tested [14, 4751], which in part are commercially distributed (e.g., ProLong and Slowfade by Molecular Probes, Mowiol by Hoechst), but the effects are far from universal, restricted to specific dyes, and often accompanied by negative side effects like a reduced brightness [14, 51], e.g., as a consequence of fluorescence quenching. The addition of CB7 provides an alternative, supramolecular approach to achieve photostabilization. Important to note, CB7 is transparent in the visible and does not act as fluorescence quencher at the relevant concentrations (mM).

The reported photobleaching quantum yields $\left(\phi_{\mathrm{b}}\right)$ of Rh6G vary between $2.5 \times 10^{-5}$ to $1.3 \times 10^{-6}[14,47-$ 49], which corresponds to a maximum of ca. 800000 
survived excitation cycles ( $\mu=1 / \phi_{\mathrm{b}}$ ) before the dye molecule becomes on average nonfluorescent through chemical transformation [49]. The photostability of Rh6G can be somewhat increased by modifying the amino group alkylation pattern, e.g., for TMR $(\mu=3$ million) and Rh123 ( $\mu=1.5$ million) [49], often by sacrificing other advantageaous fluorescence properties like a close-to-unity quantum yield. For comparison, C102 ( $\mu=2300)$ [49] is much less photostable than these rhodamines.

The strongest photostabilizing effect by $\mathrm{CB} 7$ is observed for Rh6G at high irradiance conditions, which is discussed separately [52]. Photostabilization is also significant for the remaining dyes. Addition of CB7 increases the photostability, quantified through relative photobleaching quantum yields, of PyY as well as Cy3' under high-irradiance 2nd harmonic 532-nm Nd-YAG pulsed laser excitation conditions $\left(0.1 \mathrm{~J} / \mathrm{cm}^{2}, 5-6 \mu \mathrm{M}\right.$ dye solutions) by $30 \%$, while no stabilization under high irradiance was observed for FL, which does not complex with $\mathrm{CB} 7$ and served as a negative control. The other dyes were less suitable for $532-\mathrm{nm}$ photolysis due to shifted absorption bands. Since Cy5' decomposed at least 30 times more rapidly than Rh6G under ambient light conditions in quartz cuvettes, the photostabilization provided by CB7 $(1 \mathrm{mM})$ was studied under these conditions (low irradiance). However, only a small stabilization by $10 \%$ was noticed, quantified through relative fluorescence intensities after $41 \mathrm{~h}$. The aryl ring-sulfonated Cy5, which does not form complexes with $\mathrm{CB} 7$, displayed expectedly no higher stability in the presence of $\mathrm{CB} 7$ under these low-irradiance conditions. Finally, Cy3' $(1 \mu \mathrm{M})$ was also studied at lowirradiance conditions in a photoreactor equipped with intense white light lamps, and in this case a stabilization factor of 2.1 was determined by following the absorbance, cf. Figure 2.

The photostablizing effect of $\mathrm{CB} 7$ is unquestionably related to a combination of factors, involving the inert cavity, its low polarizability, the confinement of the dye, and protection from water and oxygen. In this context one must again argue that $\mathrm{CB} 7$ complexation increases the photostability despite the higher excitedstate lifetime, since a slower radiative decay rate should allow intramolecular as well as intermolecular photoreactions to compete more efficiently. That this is not observed confirms that $\mathrm{CB} 7$ provides an active protection towards photochemical decomposition, i.e., it disfavors the chemically productive radiationless decay channels.

Complexation by macrocyclic hosts [53], akin to micellation [22-25], is expected to increase the solubility of organic solutes and to lower their tendency to adsorb to material surfaces [49] and to aggregate [54] by offering a more hydrophobic environment. In the case of the discrete complexes of macrocyclic hosts, aggregation and dimerization is also prevented by the isolation of individual dye molecules in confined environ- ments. CB7 was found to be very potent in reducing the tendency of all xanthene dyes to undergo unspecific adsorption to plastic and glass surfaces, as became directly evident from FCS count rates. Representative results of unstabilized and CB7-stabilized dye solutions with an original dye concentration of $10 \mathrm{nM}$ (obtained by dilution) demonstrated that several unstabilized dye solutions had a much lower count rate $(C R)$ than the CB7 samples (Table 2). Consequently, unless CB7 was present, a substantial amount of the dyes was lost during sample handling and preparation, which involved contact of the solutions with polypropylene and borosilicate glass surfaces. This dye loss was dramatic (up to one order of magnitude) for the xanthene dyes, suggesting a high affinity toward unspecific adsorption. From the count rates at $t=0$, the extinction coefficients at the excitation wavelength, and the fluorescence quantum yields (the last two parameters enter directly the expression for the fluorescence flow $[14,55])$ an "enhancement factor" can be calculated (Table 2), which measures how many more active dye molecules have been retained in a CB7-stabilized solution compared with an unstabilized solution. Expectedly, this number coincided, within 30\% error, with the ratio of the average numbers of molecules per irradiation area, a parameter directly obtained from the FCS experiments in the course of statistical data analysis.

In addition, the count rate in the unstabilized solutions depleted much faster (even if normalized to the different initial count rates), which revealed that unspecific adsorption continued to remove dye from solution during the ongoing microscopic measurement in the chambered cover glass. The ratio of the decrease after 30-35 min was defined as the "stabilization factor" provided by CB7 for a particular dye (Table 2); this value is only a semi-quantitative measure, strongly dependent on the storage time, materials used, etc., but along with the "enhancement factor" at $t=0$ provides the following approximate order for the adsorption propensity of the dyes: Rh6G, Rh123, PyB > PyY > TMR > Cy5'. Naturally, the "thermal" stabilization provided by CB7 is largest for those dyes, which have a high adsorption affinity, e.g., Rh6G and PyB. Among the xanthene dyes, TMR shows the smallest effects, i.e., the enhancement and stabilization due to CB7 is relatively small; this can be rationalized, since TMR possesses an additional ionizable carboxyl group to enhance water solubility. Interestingly, the cyanine dyes showed no significant difference in count rate with and without CB7, and the count rate was also quite stable over the investigated time range, suggesting that the particular cyanines are less prone to adsorb to surfaces, i.e., they are better water-soluble. The count rate data of the arylsulfonated Cy5 dye are not included in Table 2 since it does not form a complex with $\mathrm{CB} 7$, but its count rate remained also constant with time, suggesting a similar solubility as its derivative. The (unstabilized) Cy5 
Table 2. Effect of $\mathrm{CB} 7(1 \mathrm{mM})$ on the initially registered count rate (CR) and temporal depletion of count rate in $\mathrm{FCS}$ experiments for xanthene and cyanine dyes.

\begin{tabular}{lccccc}
\hline \multirow{2}{*}{ dye } & \multicolumn{2}{c}{$C R / \mathrm{kHz}^{[\mathrm{b}]}$} & $\begin{array}{c}\text { enhancement } \\
\text { factor }(t=0)\end{array}$ & $\begin{array}{c}\text { stabilization } \\
\text { factor }\end{array}$ & $\begin{array}{c}\varepsilon^{\mathrm{H}_{2} \mathrm{O}} / \varepsilon^{\mathrm{CB} 7} \\
\text { at } \lambda_{\text {exc }}\end{array}$ \\
\hline Rh6G $^{[\mathrm{a}]}$ & 13 & 96 & 12 & 100 & 1.6 \\
TMR & 3.6 & 16 & 2.6 & 1.2 & 0.79 \\
Rh123 & 8.0 & 29 & 11 & 15 & 1.4 \\
PyY & 7.4 & 39 & 4.1 & 30 & 1.0 \\
PyB & 0.8 & 19 & 12 & 40 & 1.0 \\
Cy55 $^{\prime}$ & 7.5 & 7.1 & 0.6 & 1.0 & 1.2 \\
\hline
\end{tabular}

[a] From ref. [52]. [b] Data were registered under slightly different instrumental conditions, e.g., laser irradiance, and are not accurately comparable between different dyes; 514-nm Ar/2 laser irradiation, except for Cy5' (633-nm HeNe-2 laser); 10\% error. [c] Calculated as $\left(C R_{t=0}^{\mathrm{CB} 7} /\left(\varepsilon^{\mathrm{CB} 7} \Phi_{\mathrm{f}}^{\mathrm{CB} 7}\right)\right) /\left(C R_{t=0}^{\mathrm{H}_{2} \mathrm{O}} /\left(\varepsilon^{\mathrm{H}_{2} \mathrm{O}} \Phi_{\mathrm{f}}^{\mathrm{H}_{2} \mathrm{O}}\right)\right)$. [d] Calculated as $\left(1-\left(C R^{\mathrm{H}_{2} \mathrm{O}} / C R_{t=0}^{\mathrm{H}_{2} \mathrm{O}}\right)\right) /\left(1-\left(C R^{\mathrm{CB} 7} / C R_{t=0}^{\mathrm{CB} 7}\right)\right)$ after 30-35 min FCS measurement time. The stabilization factor defined in this way reflects how much more dye has become inactive (presumably due to adsorption) in the absence than in the presence of CB7 in the course of the FCS measurement.

dye showed also the largest count rate in the FCS experiments (ca. $125 \mathrm{kHz}$ ), followed by the Rh6G solution stabilized with CB7 (96 kHz), while those in the remaining unstabilized solutions were one order of magnitude lower (Table 2).

The possibility to stabilize the various dyes thermally as well as photochemically is of interest for a range of applications, including dye lasers, FCS, as quantum counters and spectral references, and scanning confocal microscopy. In addition, the administration of CB7 should allow a long-term storage of more concentrated as well as very dilute dye solutions, such that the often emphasized fresh preparation of samples [50] may no longer be necessary for selected applications, e.g., in order to use a particular dye as a diffusioncoefficient or count rate reference in FCS. For example, a CB7-stabilized aqueous solution of Rh6G in a borosilicate glass vial could be kept over several weeks under ambient light at a window without significant depletion of the absorption and fluorescence characteristics [52].

In summary, the addition of CB7 to aqueous solutions of some of the most important cationic fluorescent dyes displays a whole range of advantageous effects, which include increased quantum yields, increased fluorescence lifetimes, spectral shifts, increased photostability and prevention of association and aggregation. These are of interest for a whole latitude of practical applications, including the substitution of organic solvents by water, which assists the development of a "green dye chemistry" as well as environmental applications. The stabilization of the dyes is also expected when the dyes are attached to biomolecules, since the complexation of a fluorophore by $\mathrm{CB} 7$ in a labelled peptide has recently been reported [11]. Additional applications of CB7 for a variety of biochemical applications, including assays for high-throughput screening or PCR, will therefore have to be explored. The use of solid-state materials based on photostable cucurbituril complexes of organic dyes is more far fetched, but of current interest since the next generation of data storage media is anticipated to be based on fluorescence rather than optical reflection.

\section{EXPERIMENTAL SECTION}

The fluorescent dyes were used as received from Molecular Probes (Rh6G), Fluka (Rh123, TMR, CV, FL, C102), Aldrich (PyB and PyY), FEW Chemicals (Cy3' and Cy5'), Amersham Biosciences (Cy5), and Bfi OPTiLAS (IR140 and IR144). The optimized synthetic procedure for CB7 has been published previously [11].

The pulsed laser irradiations of dye solutions with and without CB7 ( $1 \mathrm{mM})$ were carried out by using the 2nd harmonic (532 nm) output of a Nd-YAG laser (Continuum Surelite III 10 model, ca. $0.1 \mathrm{~J}$ per pulse). The photostabilization of the dyes (ca. 1-10 $\mu \mathrm{M}$, OD ca. 0.3 ) was monitored through the fluorescence intensity with a Varian Cary Eclipse fluorometer or through the characteristic visible absorption band using a Varian Cary 4000 UV-Vis spectrophotometer relative to the optically matched unstabilized dye solutions. For quantification, the decrease of absorbance at $532 \mathrm{~nm}(A)$ at different irradiation times was plotted according to the pertinent function $\log \left(\left[10^{A} 0-1\right] /\left[10^{A}-1\right]\right)$ [56]. The photobleaching quantum yield was determined from the ratio of the slopes $S^{\mathrm{H}_{2} \mathrm{O}} / S^{\mathrm{CB} 7}=\varepsilon^{\mathrm{H}_{2} \mathrm{O}} \Phi_{\mathrm{b}}^{\mathrm{H}_{2} \mathrm{O}} /\left(\varepsilon^{\mathrm{CB} 7} \Phi_{\mathrm{b}}^{\mathrm{CB} 7}\right)$, where ${ }_{\varepsilon}^{\mathrm{CB} 7}$ and $\varepsilon^{\mathrm{H}_{2} \mathrm{O}}$ are the extinction coefficients of the dye with and without $\mathrm{CB} 7$, respectively, at the irradiation wavelength of $532 \mathrm{~nm}$. The stabilization factors stated in the text are given as the ratio of the photobleaching quantum yields in the absence and presence of the stabilizer. Alternatively, the decrease in the absorbance and fluorescence intensity of the stabilized and unstabilized dye solutions was followed under daylight irradiation (ambient conditions), or upon irradiation in a Luzchem LZC-4V photoreactor equipped with $14 \mathrm{cool}$ white fluorescent tubes; in these cases, no corrections for differential extinction coefficients were made. 
The photobleaching of very dilute dye solutions $(10 \mathrm{nM})$ with and without $\mathrm{CB} 7(1 \mathrm{mM})$ was also monitored by FCS on a confocal microscope (Carl Zeiss LSM 510 Confocor 2). The dye solutions were irradiated with intensity-adjusted 514-nm laser light (30 mW) from a CW Ar/2 laser or 633-nm laser light (6 mW) from a HeNe-2 laser. As sample container, a Lab-Tek 8-chambered borosilicate cover glass with polystyrene chamber walls was employed. The decrease in the count rate $(\mathrm{CR})$, which is directly proportional to the average fluorescence intensity of the sample, was measured with increasing measurement time. For the determination of the enhancement and stabilization factors in Table 2, refer to the table footnotes.

The fluorescence lifetimes of low micromolar dye solutions in the presence and absence of $\mathrm{CB} 7$ were measured by time-correlated single-photon-counting (TCSPC) on an FLS-920 fluorometer (Edinburgh Instruments) using a $\mathrm{H}_{2}$-flash lamp as excitation source. The fluorescence quantum yields of the CB7-stabilized dyes were estimated by comparing the integrated corrected fluorescence spectra with those of the unstabilized dyes, for which the quantum yields are known (see Table 1 for references). The excitation wavelengths were selected to achieve optical matching of the stabilized and unstabilized dye solutions at low OD values $(\leq 0.1)$. The photophysical parameters (absorption and fluorescence maxima, fluorescence quantum yields and lifetimes) reported herein for water as solvent are in good agreement with literature data for Rh6G [40], TMR [49, 57], Rh123 [49], C102 [41, 49], CV [58], Cy5 [59], and FL [40].

\section{ACKNOWLEDGMENTS}

This work was supported by the International University Bremen. We would like to thank A. L. Koner for the help with the photobleaching experiments.

\section{REFERENCES}

[1] B. D. Wagner, ed., Fluorescence Studies of Supramolecular Host-Guest Inclusion Complexes, American Scientific Publishers, Stevenson Ranch, CA, 2003, Vol. 3, pp 1-57.

[2] S. Hamai and A. Nakamura, eds., Inclusion Complexes of Cyclodextrins in Aqueous Solution, American Scientific Publishers, Stevenson Ranch, CA, 2003, Vol. 3, pp. 59-119.

[3] P. Bortulus and S. Monti, Adv. Photochem. 21 (1996), 1-133.

[4] W. L. Mock, Comprehensive Supramolecular Chemistry (F. Vögtle, ed.), Elsevier, New York, 1996, Vol. 2, pp 477-493.

[5] J. W. Lee, S. Samal, N. Selvapalam, H.-J. Kim, and K. Kim, Acc. Chem. Res. 36 (2003), 621-630.
[6] H.-J. Buschmann and T. Wolff, J. Photochem. Photobiol. A: Chemistry 121 (1999), 99-103.

[7] B. D. Wagner and A. I. MacRae, J. Phys. Chem. B 103 (1999), 10114-10119.

[8] B. D. Wagner, S. J. Fitzpatrick, M. A. Gill, A. I. MacRae, and N. Stojanovic, Can. J. Chem. 79 (2001), 1101-1104.

[9] S. Y. Jon, N. Selvapalam, D. H. Oh, J.-K. Kang, S.-Y. Kim, Y. J. Jeon, J. W. Lee, and K. Kim, J. Am. Chem. Soc. 125 (2003), 10186-10187.

[10] C. Marquez and W. M. Nau, Angew. Chem. 113 (2001), 4515-4518; Angew. Chem. Int. Ed. 2001, 4540, 4387-4390.

[11] C. Marquez, F. Huang, and W. M. Nau, IEEE Trans. Nanobiosci. 3 (2004), 39-45.

[12] J. Mohanty and W. M. Nau, Photochem. Photobiol. Sci. 3 (2004), 1026-1031.

[13] M. A. Rankin and B. D. Wagner, Supramol. Chem. 16 (2004), 513-519.

[14] C. Eggeling, J. Widengren, R. Rigler, and C. A. M. Seidel, Photostability of Fluorescent Dyes for SingleMolecule Spectroscopy: Mechanism and Experimental Methods for Estimating Photobleaching in Aqueous Solution, Springer-Verlag, 1999.

[15] C. Zander, M. Sauer, K. H. Drexhage, D.-S. Ko, A. Schulz, J. Wolfrum, L. Brand, C. Eggeling, and C. A. M. Seidel, Appl. Phys. B 63 (1996), 517-523.

[16] J. Slavik, Fluorescent Probes in Cellular and Molecular Biology, CRC Press, Boca Raton, 1994.

[17] M. Heupel, I. Gregor, S. Becker, and E. Thiel, International J. of Photoenergy 1 (1999), 165-172.

[18] S. Schultheiss, E. Yariv, R. Reisfeld, and H. D. Breuer, Photochem. Photobiol. Sci. 1 (2002), 320323.

[19] K. Hara, K. Sayama, Y. Ohga, A. Shinpo, S. Suga, and H. Arakawa, Chem. Commun. (2001), 569-570.

[20] S. K. Grant, J. G. Sklar, and R. T. Cummings, J. Biomol. Screen 7 (2002), 531.

[21] M. Kawakami, K. Koya, T. Ukai, N. Tatsuta, A. Ikegawa, K. Ogawa, T. Shishido, and L. B. Chen, J. Med. Chem. 41 (1998), 130-142.

[22] R. Humphry-Baker, M. Grätzel, and R. Steiger, J. Am. Chem. Soc. 102 (1980), 847-848.

[23] J. C. Mialocq, M. Meyer, P. Hébert, X. Armand, and D. Lambert, Opt. Commun. 77 (1990), 185-191.

[24] J. C. Mialocq, P. Hébert, X. Armand, R. Bonneau, and J. P. Morand, J. Photochem. Photobiol. A: Chem. 56 (1991), 323-338.

[25] O. G. Peterson, S. A. Tuccio, and B. B. Snavely, Appl. Phys. Lett. 17 (1970), 245-247.

[26] T. V. S. Rao, J. B. Huff, and C. Bieniarz, Tetrahedron 54 (1998), 10627-10634.

[27] A. Mishra, R. K. Behera, P. K. Behera, B. K. Mishra, and G. B. Behera, Chem. Rev. 100 (2000), 19732011.

[28] Y. Liu, B.-H. Han, and Y.-T. Chen, J. Phys. Chem. B 106 (2002), 4678-4687. 
[29] I. R. Politzer, K. T. Crago, T. Hampton, J. Joseph, J. H. Boyer, and M. Shah, Chem. Phys. Lett. 159 (1989), 258-262.

[30] B. Reija, W. Al-Soufi, M. Novo, and J. V. Tato, J. Phys. Chem. B 109 (2005), 1364-1370.

[31] J. E. H. Buston, J. R. Young, and H. L. Anderson, Chem. Commun. (2000), 905-906.

[32] J. Kim, I.-S. Jung, S.-Y. Kim, E. Lee, J.-K. Kang, S. Sakamoto, K. Yamaguchi, and K. Kim, J. Am. Chem. Soc. 122 (2000), 540-541.

[33] C. Marquez, R. R. Hudgins, and W. M. Nau, J. Am. Chem. Soc. 126 (2004), 5806-5816.

[34] J. Mohanty and W. M. Nau, unpublished results.

[35] T. Wohland, K. Friedrich, R. Hovius, and H. Vogel, Biochemistry 38 (1999), 8671-8681.

[36] K. Modos, R. Galantai, I. Bardos-Nagy, M. Wachsmuth, K. Toth, J. Fidy, and J. Langowski, Eur. Biophys. J. 33 (2004), 59-67.

[37] B. Acemioglu, M. Arik, and Y. Onganer, J. Lumin. 97 (2002), 153-160.

[38] K. H. Drexhage, T. W. Hansch, E. P. Ippen, F. P. Schafer, C. V. Shank, and B. B. Snavely, Dye Lasers, Springer-Verlag, Berlin, 1973.

[39] A. S. Tatikolov and S. M. B. Costa, Photochem. Photobiol. Sci. 1 (2002), 211-218.

[40] D. Magde, R. Wong, and P. G. Seybold, Photochem. Photobiol. 75 (2002), 327-334.

[41] G. Jones II, W. R. Jackson, C.-Y. Choi, and W. R. Bergmark, J. Phys. Chem. 89 (1985), 294-300.

[42] S. J. Strickler and R. A. Berg, J. Chem. Phys. 37 (1962), 814-822.

[43] I. Hemmilä and S. Webb, Drug. Discov. Today 2 (1997), 373-381.
[44] J. A. Smith, R. M. West, and M. Allen, J. Fluoresc. 14 (2004), 151-171.

[45] C. Marquez, U. Pischel, and W. M. Nau, Org. Lett. 5 (2003), 3911-3914.

[46] K. Suhling, P. M. W. French, and D. Phillips, Photochem. Photobiol. Sci. 4 (2005), 13-22.

[47] T. Schmidt, G. J. Schütz, W. Baumgartner, H. J. Gruber, and H. Schindler, J. Phys. Chem. 99 (1995), 17662-17668.

[48] L. A. Deschenes and D. A. V. Bout, Chem. Phys. Lett. 365 (2002), 387-395.

[49] C. Eggeling, J. Widengren, R. Rigler, and C. A. M. Seidel, Anal. Chem. 70 (1998), 2651-2659.

[50] P. S. Dittrich and P. Schwille, Appl. Phys. B 73 (2001), 829-837.

[51] R. J. Florijn, J. Slats, H. J. Tanke, and A. K. Raap, Cytometry 19 (1995), 177-182.

[52] J. Mohanty and W. M. Nau, Angew. Chem., in press.

[53] J. Szejtli, Chem. Rev. 98 (1998), 1743-1753.

[54] O. Valdes-Aguilera and D. C. Neckers, Acc. Chem. Res. 22 (1989), 171-177.

[55] S. T. Hess and W. W. Webbdagger, Biophys. J. 83 (2002), 2300-2317.

[56] W. Adam, G. Fragale, D. Klapstein, W. M. Nau, and J. Wirz, J. Am. Chem. Soc. 117 (1995), 12578-12592.

[57] S. A. Soper, H. L. Nutter, R. A. Keller, A. Richard, L. M. Davis, and E. B. Shera, Photochem. Photobiol. 57 (1993), 972-977.

[58] S. J. Isak and E. M. Eyring, J. Phys. Chem. 96 (1992), 1738-1742.

[59] R. B. Mujumdar, L. A. Ernst, S. R. Mujumdar, C. J. Lewis, and A. S. Waggoner, Bioconj. Chem. 4 (1993), 105-111. 


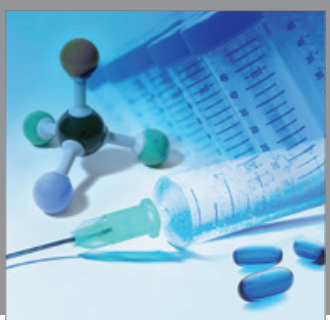

International Journal of

Medicinal Chemistry

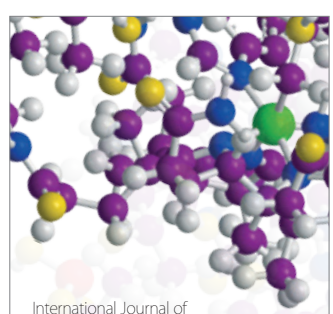

Carbohydrate Chemistry

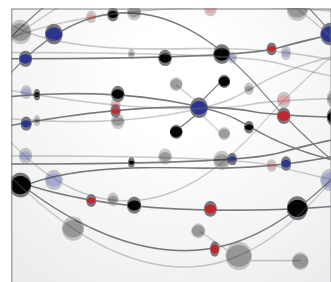

The Scientific World Journal
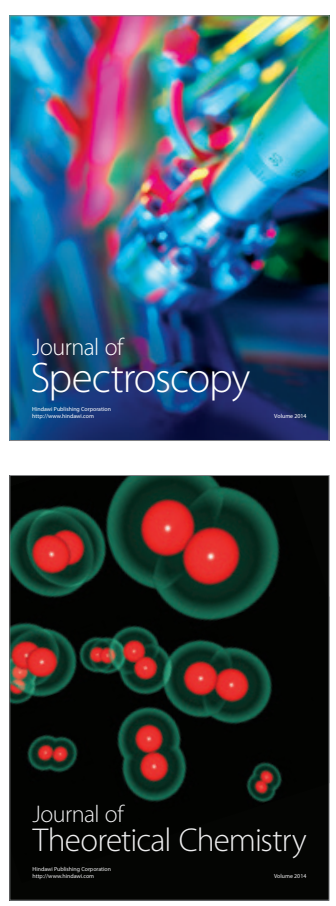
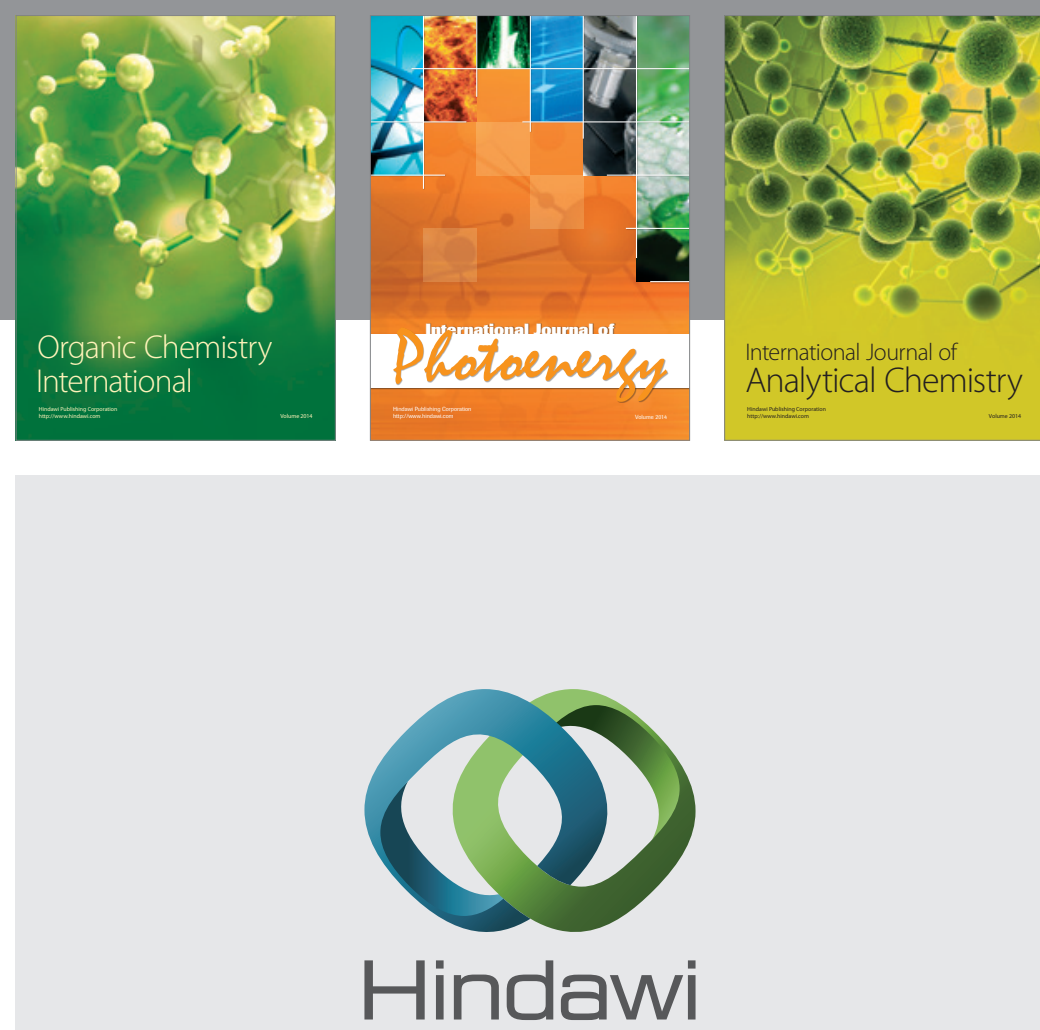

Submit your manuscripts at

http://www.hindawi.com
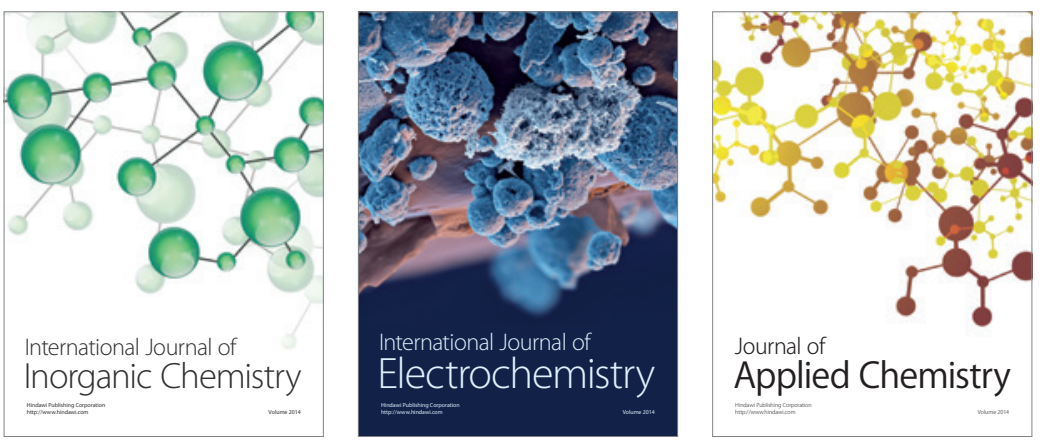

Journal of

Applied Chemistry
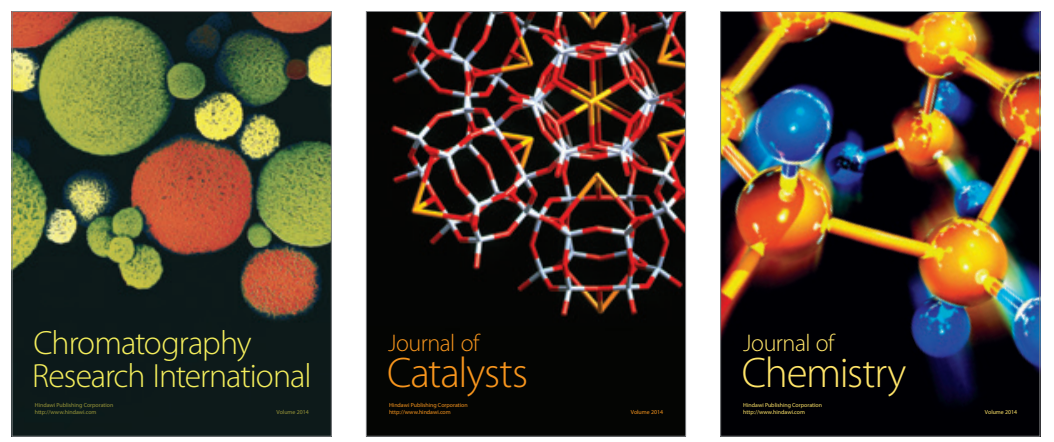
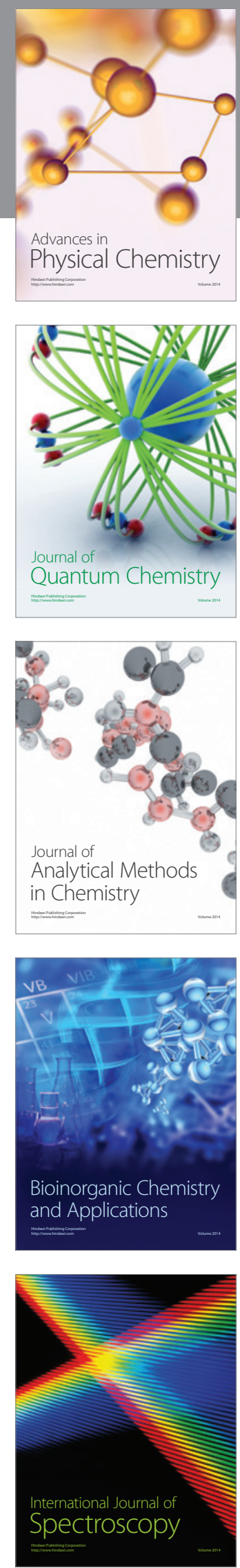